\title{
Timing Neurogenesis and Differentiation: Insights from Quantitative Clonal Analyses of Cerebellar Granule Cells
}

\author{
J. Sebastian Espinosa and Liqun Luo \\ Howard Hughes Medical Institute and Department of Biology, Neurosciences Program, Stanford University, Stanford, California 94305
}

\begin{abstract}
The cerebellum is an excellent model system to study how developmental programs give rise to exquisite neuronal circuits in the adult brain. Here, we describe our findings regarding granule cell neurogenesis and differentiation using the MADM method (mosaic analysis with double markers) in mice. By following the development of individual granule cell clones, we show that (1) granule cell precursors (GCPs) undergo predominantly symmetric division during postnatal development; (2) clonally related granule cells (GCs) exit the cell cycle within a narrow time window and stack their axons in the molecular layer in chronological order from deep to superficial sublayers; and (3) whereas the average GCP proliferation in the external granular layer is progressively slower as development proceeds, there is a rapid expansion of GCPs shortly before clonally related GCs exit the cell cycle. These properties produce GC clones that are distinct, each having a restricted axonal projection, but that are on average similar in cell number. We discuss possible developmental mechanisms and functional implications of these findings.
\end{abstract}

Key words: cerebellum; cell lineage; granule cells; clonal analysis; neurogenesis; MADM

\section{Introduction}

The small number of cell types that contribute to the crystallinelike organization makes the cerebellar cortex an attractive system to study fundamental principles of neural development, organization, function, and disease (Ramon y Cajal, 1911; Eccles, 1970; Ito, 1984; Herrup and Kuemerle, 1997; Wang and Zoghbi, 2001; Sillitoe and Joyner, 2007). The granule cell (GC) is a major cell type in the cerebellar cortex, accounting for as many as half of all neurons in the CNS (Fox and Barnard, 1957). GCs originate from a germinal zone in the upper rhombic lip (Miale and Sidman, 1961; Alder et al., 1996). In mice, GC precursors (GCPs) leave the rhombic lip around embryonic day 13 (E13) and migrate rostrally over the surface of the cerebellar anlage to form a mitotically active region called the external granular layer (EGL) (Hatten and Heintz, 1995). The EGL starts to form at E15, and GCPs within the EGL remain mitotically active until the third postnatal week (Miale and Sidman, 1961; Hanaway, 1967). Around the time of birth, some GCPs start to exit the cell cycle and begin to differentiate into mature GCs resulting in the gradual disappearance of the EGL. GCs first extend axons parallel to the pial surface and then migrate radially along Bergmann glia to populate the internal granular layer (IGL), the final position of GCs (Rakic,

Received Nov. 20, 2007; accepted Jan. 14, 2008.

This work was supported by National Institutes of Health Grant R01-NS050835 to L.L. J.S.E. is a predoctoral National Research Service Award fellow. L.L. is a Howard Hughes Medical Institute investigator. We are grateful to colleagues for advice and critical reading of this manuscript, in particular Alexandra Joyner, Emilie Legue, Ben Barres, Susan McConnell, and Mark Schnitzer; and to members of the Luo laboratory, in particular Hui Zong, Mandar Muzumdar, Bosiljka Tasic, Simon Hippenmeyer, Kazunari Miyamichi, and Ling Li. We thank Corrine Lobe for $\beta$-ActinCreER mice and Joe Zhong for technical assistance.

Correspondence should be addressed to Dr. Liqun Luo, Department of Biology, Stanford University, Stanford, CA 94305. E-mail: lluo@stanford.edu.

DOI:10.1523/JNEUROSCI.5157-07.2008

Copyright $\odot 2008$ Society for Neuroscience $\quad$ 0270-6474/08/282301-12\$15.00/0
1971; Adams et al., 2002). The IGL becomes obvious at postnatal day 5 (P5) and is completed by P20 (Altman and Bayer, 1997).

Lineage analysis can elucidate the organizational principles of development by providing information about the origin of cell types. In the context of GC development, transplantation experiments in chick (Hallonet et al., 1990) and retroviral labeling in rat (Zhang and Goldman, 1996) have demonstrated that the EGL only gives rise to a single cell type, the GCs. Fate mapping studies elegantly showed that all GCs can be traced back to a subset of Math1-expressing cells in the rhombic lip (Machold and Fishell, 2005; Wang et al., 2005). Genetic and viral-mediated fatemapping approaches further delineate the organizational relationship between cells in the rhombic lip and their final destinations in the cerebellar cortex, as well as their migration patterns (Ryder and Cepko, 1994; Mathis and Nicolas, 2003; Sgaier et al., 2005). However, these studies have not followed the progression of clonally related GCs to adult to examine the relationship between lineage and axonal wiring patterns.

We recently developed a mosaic analysis method termed MADM (mosaic analysis with double markers) that permits lineage tracing with the ability to visualize axonal and dendritic projections of all progeny. By applying this method, we found that GCs originating from single GCPs as early as E14 restrict their axons to a sublayer in the molecular layer (ML) although the cell bodies are dispersed throughout the IGL (Zong et al., 2005). Here, we explore the underlying mechanisms by following the development of individual GC clones in conjunction with their axonal projection patterns. We uncover new and unexpected findings regarding GC neurogenesis and differentiation.

\section{Materials and Methods}

Generation of MADM mice. RG and GR mice were separately crossed with $\beta$-Actin-CreER mice (Guo et al., 2002). Double-positive GR/+; $\beta$-Actin- 
Table 1. Summary of labeling frequency in different experimental paradigms

\begin{tabular}{|c|c|c|c|c|c|c|c|c|}
\hline \multirow[b]{2}{*}{ Tamoxifen } & \multirow[b]{2}{*}{ CldU } & \multicolumn{7}{|c|}{ Age of brain dissection } \\
\hline & & P3 & P6 & P9 & $\mathrm{P} 12$ & P15 & P18 & P21 \\
\hline E17.5 (8 mg) & & $5 B / 9 Y$ & 20B/34Y/9GR & $19 \mathrm{~B} / 55 \mathrm{Y} / 5 \mathrm{GR}$ & 40B/108Y/21GR & 20B/58Y/8GR & $14 \mathrm{~B} / 34 \mathrm{Y} / 4 \mathrm{GR}$ & $38 \mathrm{~B} / 83 \mathrm{Y} / 5 \mathrm{GR}$ \\
\hline E17.5 (8 mg) & P6 & & & & & & & 11B/15Y \\
\hline E17.5 (8 mg) & P9 & & & $8 \mathrm{~B} / 28 \mathrm{Y}$ & & & & \\
\hline $\mathrm{E} 17.5(8 \mathrm{mg})$ & P15 & & & & & & & $9 \mathrm{~B} / 25 \mathrm{Y}$ \\
\hline P6 (1 mg) & P6 & & & & & & & $9 \mathrm{~B} / 45 \mathrm{SC}$ \\
\hline P15 (4 mg) & P15 & & & & & & & $8 \mathrm{~B} / 47 \mathrm{SC}$ \\
\hline P3 $(0.5 \mathrm{mg})$ & & & & & & & & $3 \mathrm{~B} / 7 \mathrm{GR}$ \\
\hline P6 (1 mg) & & & & & & & & $12 \mathrm{~B} / 7 \mathrm{GR}$ \\
\hline P9 (4 mg) & & & & & & & & $6 \mathrm{~B} / 8 \mathrm{GR}$ \\
\hline P12 (4 mg) & & & & & & & & 16B/6GR \\
\hline
\end{tabular}

B, Brains; $Y, G_{2}-Z$ or $G_{1}$ clones analyzed; $G R, G_{2}-X$ clones analyzed; $S C$, single cells analyzed. Note that all clones generated from E17.5 tamoxifen injections are included in the above analysis. Not all clones and single cells for postnatal tamoxifen injections are included in the above analysis.

CreER/+ and RG/+; $\beta$-Actin-CreER/+ mice were kept as separate stocks before MADM analysis and were genotyped as described previously (Zong et al., 2005). These mice were crossed to each other to generate GR/RG; $\beta$-Actin-CreER/+ or GR/RG; $\beta$-Actin-CreER/ $\beta$-Actin-CreER mice for MADM analysis. The presence of the Cre transgene was determined as described previously (Zong et al., 2005). We do not distinguish progeny that are homozygous or heterozygous for $\beta$-Actin-CreER.

Clonal analysis in the cerebellum. Experimental conditions are summarized in Table 1. Tamoxifen (T-5648; Sigma, St. Louis, MO) was dissolved in corn oil (C-8267; Sigma) at a concentration of $20 \mathrm{mg} / \mathrm{ml}$ for $\sim 4$ $\mathrm{h}$ at room temperature, stored at $4^{\circ} \mathrm{C}$ covered with aluminum foil, and used within 2 weeks. To induce interchromosomal recombination in GR/RG; $\beta$-Actin-CreER/+ or GR/RG; $\beta$-Actin-CreER/ $\beta$-Actin-CreER pups, tamoxifen was administered by intraperitoneal injection. Controls were given corn oil without tamoxifen [ 12 of 12 brains with no green fluorescent protein (GFP) labeling]. The probability that a cluster of GCs is derived from two independent but overlapping clones was estimated to be $4.4 \%$ for $8 \mathrm{mg}$ tamoxifen injections at E17.5 (Zong et al., 2005). Sixty-micrometer-thick serial sagittal sections of cerebella were stained and imaged. High-resolution MADM labeling allows us to easily identify cell type by characteristic GC morphology and positioning of cell bodies. Clonal types [EGL, mix of EGL/ML/IGL (MIX), and IGL] were determined using 4',6-diamidino-2-phenylindole (DAPI) as a counterstain and the morphology of cells (polyhedral, nonpolyhedral, axonal, and dendritic processes). IGL clonal types (deep, middle, superficial projecting) were classified based on average axonal projections to the bottom one-third, middle one-third, or top one-third of the ML.

Tissue preparation and histochemistry. All animal procedures were based on animal care guidelines and were approved by the Stanford University Administrative Panels on Laboratory Care. For histology, mice were anesthetized with an intraperitoneal injection of $2.5 \%$ Avertin (400-600 mg/kg body weight) and perfused with $0.1 \mathrm{M} \mathrm{PBS,} \mathrm{pH} 7.4$, followed by ice-cold $4 \%$ paraformaldehyde (PFA) in $0.1 \mathrm{~m}$ PBS. Tissues were isolated and fixed in $4 \% \mathrm{PFA}$ at $4^{\circ} \mathrm{C}$ overnight, washed three times in PBS, cryoprotected for $24 \mathrm{~h}$ in $30 \%$ sucrose in PBS, and embedded in Tissue-Tek OCT before cryostat sectioning. Cryosections ( $60 \mu \mathrm{m}$ thick) were washed thee times for $10 \mathrm{~min}$ in PBS, blocked with $10 \%$ normal donkey serum (NDS) in 0.1 m PBS plus $0.3 \%$ Triton X-100 (PBT) for 60 min, and stained at $4^{\circ} \mathrm{C}$ overnight with rabbit anti-GFP (1:500; Invitrogen, Eugene, OR) and preabsorbed goat anti-MYC (1:200; Novus, Littleton, CO). After PBT washes, the sections were stained at $4^{\circ} \mathrm{C}$ overnight or for $2 \mathrm{~h}$ at room temperature with donkey anti-rabbit Alexa 488 (1:200; Invitrogen) and donkey anti-goat Cy3 (1:500; Jackson ImmunoResearchWest Grove, PA). After four 10 min washes in PBT, including in the last wash the DNA dye DAPI for $10 \mathrm{~min}$, samples were mounted in Gel/Mount mounting medium (Biomeda, Foster City, CA). Preabsorption of goat anti-MYC (see above) was conducted by postfixing three wild-type brains cut into small pieces, blocked at $4^{\circ} \mathrm{C}$ overnight with $10 \%$ NDS in PBT, and transferred into $30 \mathrm{ml}$ of working solution goat antiMYC in PBT for a $2 \mathrm{~d}$ absorption. Images were taken by $1 \mu \mathrm{m}$ optical sectioning using Zeiss (Oberkochen, Germany) confocal microscopy.

5-Chloro-2-deoxyuridine and phospho-histone 3 analysis. See Table 1 for specific conditions. Solutions of 5-chloro-2-deoxyuridine (CldU) were prepared at $17 \mathrm{mg} / \mathrm{ml}$ in sterile PBS and intraperitoneally injected at $2.5 \mathrm{ml} / \mathrm{kg}$ to achieve an effective dose of $42.5 \mathrm{mg} / \mathrm{kg}$ and the same molar concentration as previously described for bromodeoxyuridine (BrdU) (Gage et al., 1995; Vega and Peterson, 2005). Thymidine analog solution was administered by intraperitoneal injection to ensure precise delivery. After detection of MADM clones in 30- $\mu \mathrm{m}$-thick sections (see above, Tissue preparation and histochemistry; donkey anti-goat Cy5 was used instead of donkey anti-goat Cy3; 1:200; Jackson ImmunoResearch), tissue was permeabilized with $\mathrm{HCl} 2 \mathrm{~N}$ in $\mathrm{PBS}$ for $30 \mathrm{~min}$ at $37^{\circ} \mathrm{C}$, washed three times with PBS, blocked with $10 \%$ NDS in PBT, and stained at $4^{\circ} \mathrm{C}$ overnight with rat anti-BrdU (1:1000; Accurate \#OBT-0030; clone BU1/ 75). After PBT washes, the sections were stained at $4^{\circ} \mathrm{C}$ overnight or for $2 \mathrm{~h}$ at room temperature with Cy3-conjugated donkey anti-rat (1:200; Jackson ImmunoResearch). Detection of phospho-histone 3 (pH3) was accomplished using rabbit anti-pH3 (Ser-10; 1:250; Upstate Biotechnology, Lake Placid, NY) and donkey anti-rabbit Cy3 (1:200; Jackson ImmunoResearch Laboratories) in conjunction with detection of MADM clones (see above, Tissue preparation and histochemistry; donkey antigoat $\mathrm{Cy} 5$ was used instead of donkey anti-goat Cy3). All MADM clones in these analyses were double positive for GFP and MYC. For experiments involving coinjection of tamoxifen and CldU at postnatal time points, GCs were quantified only when the axonal fiber could be traced to its T-bifurcation in the ML. In some cases, sagittal sections at the lateral extremities showed longer T-bifurcations because of the plane of sectioning.

\section{Results}

\section{GCPs expand predominantly via symmetric cell division}

The mode of neural precursor division has important implications for patterns of neurogenesis. In the cerebral cortex, for instance, neural precursors in the ventricular zone undergo characteristic changes of division pattern from early asymmetric division to later symmetric division (Langman et al., 1966; Martin, 1967; Chenn and McConnell, 1995; Zhong et al., 1996). What mode of division is used for GC neurogenesis has not been analyzed previously. Do GCPs undergo symmetric cell division and exponential expansion, asymmetric cell division and linear expansion, or a combination of the two? Does the cell division mode change over time? Analysis of $\mathrm{MADM} \mathrm{G}_{2}-\mathrm{X}$ events can be used to resolve these issues, because sister cells generated after $G_{2}$ recombination followed by $\mathrm{X}$ segregation $\left(\mathrm{G}_{2}-\mathrm{X}\right.$ event) would be labeled by two different colors (Fig. 1A) (Zong et al., 2005). A symmetric division model predicts that green and red cells that originate from the same precursor should be present in equal numbers, whereas an asymmetric division model predicts that clones are predominantly of one color (Fig. $1 B$ ). In addition, the time of clone induction can be used to determine the birth date of GCPs giving rise to the clones.

We used the $\beta$-Actin-CreER transgene (Guo et al., 2002) to 
A

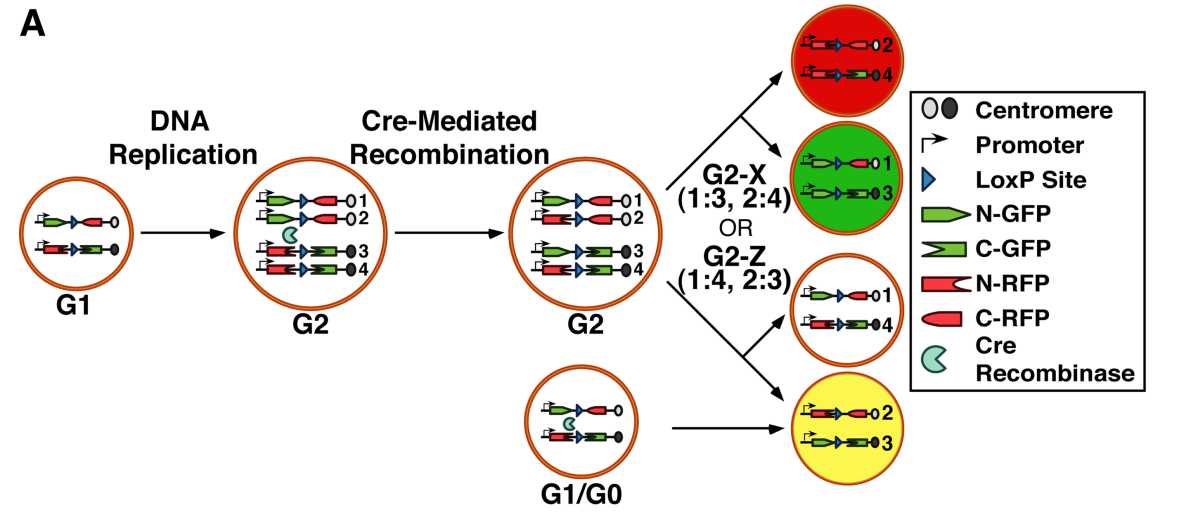

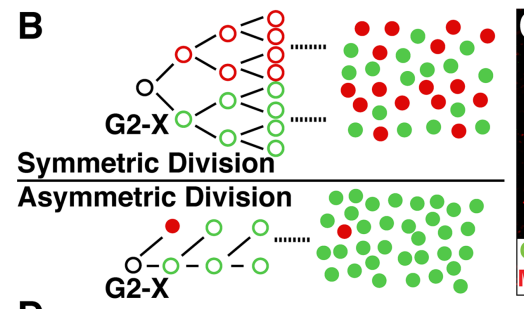

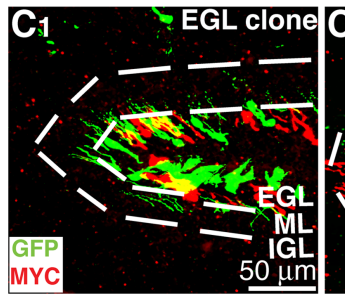

D
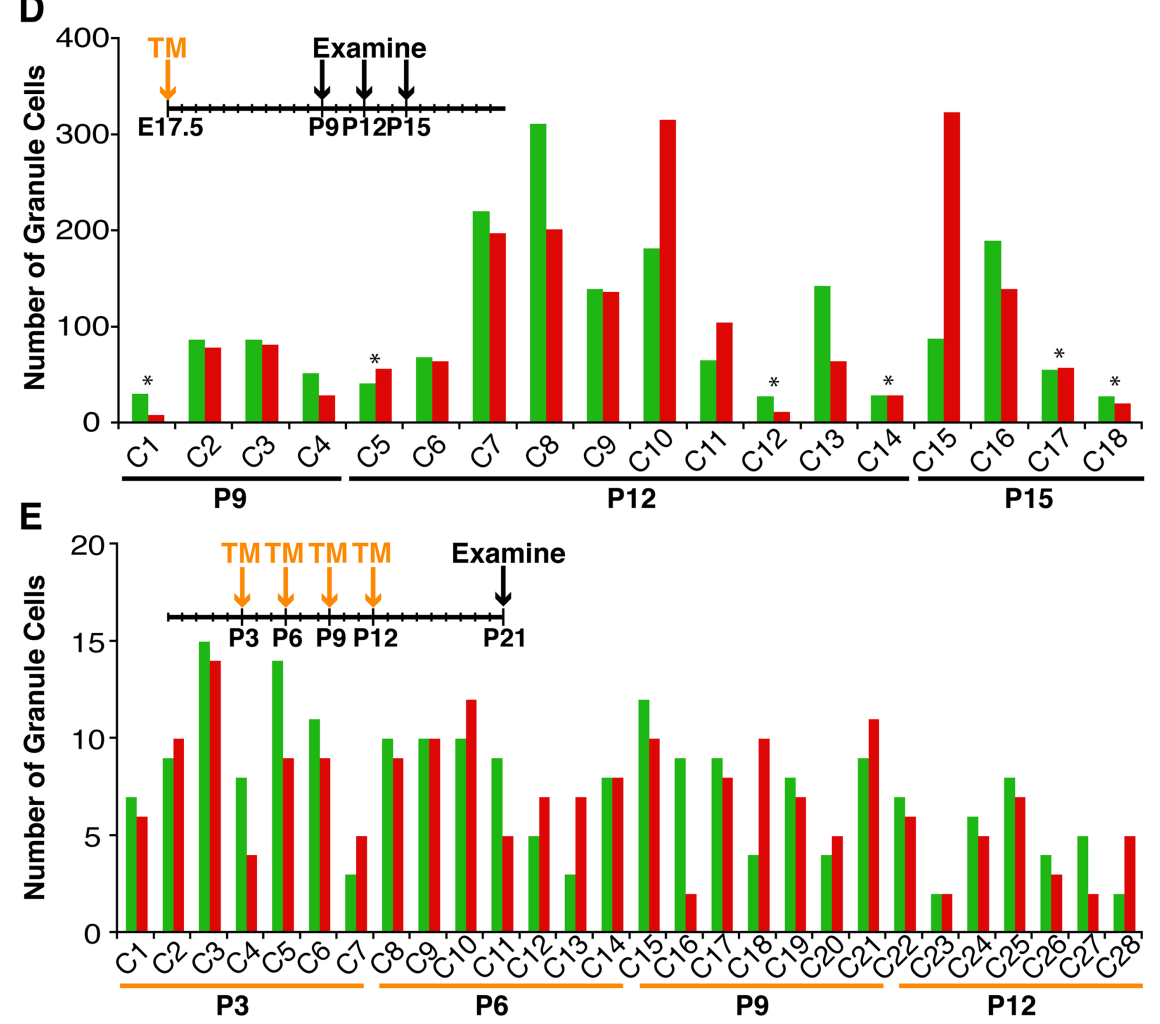

Figure 1. GCPs divide predominantly symmetrically during perinatal and postnatal development. $A$, Schematic of the MADM method illustrating (re-mediated interchromosomal recombination that results in reconstitution of two fluorescent markers, GFP and red fluorescent protein (dsRed2-Myc). If recombination occurs in $G_{2}$ phase, chromatids can segregate to generate two differentially labeled cells $\left(G_{2}\right.$-Xsegregation), or one doubly labeled cell and one unlabeled cell ( $G_{2}$-Z segregation). Recombination in $\mathrm{G}_{1}$ or $\mathrm{G}_{0}$ generates a doubly labeled cell. $B, \mathrm{BADM}_{2}$-X labeling can distinguish between two modes of division: symmetric and asymmetric. Completely symmetric divisions should generate same numbers of differentially labeled cells, whereas completely asymmetric divisions should generate a single cell labeled with one marker and all other cells labeled with the other marker. Open circles, GCPs; filled circles, postmitotic GCS. C, Two exemplary confocal images of MADM G ${ }_{2}-$ X labeling of GCs. Tamoxifen (TM) was applied intraperitoneally at E17.5 in the pregnant mother. Progeny of the genotype GR/RG; $\beta$-Actin-CreER were examined. $C_{1}, A$ clone of dividing GCPs restricted to the EGL. $C_{2}$, A clone of differentiated GCS restricted to the IGL. Dashed white lines trace the borders between layers based on DAPI staining not shown. $\boldsymbol{D}$, Inset, Schematic of the experiment (TM application at E17.5; dissection time points at P9-P15). The bar graph shows paired counts for green and red cells in each clone analyzed. The asterisk represents EGL clones, which are markedly smaller than IGL clones (the rest) that have completed cell division (see Fig. 4). We exclude $G_{2}-X$ clones $<25$ cells because a lower total number has less power, although small clones also followed this trend with a similar number of red and green cells. $\boldsymbol{E}$, Inset, Schematic of the experiment (TM application at P3-P12; dissection at P21). The bar graph shows paired counts for green and red cells in each GC clone analyzed. induce $\mathrm{G}_{2}-\mathrm{X}$ MADM clones at E17.5. In the absence of tamoxifen, CreER protein is sequestered in the cytoplasm and therefore cannot catalyze DNA recombination. When CreER binds tamoxifen, it translocates into the nucleus where Cre can catalyze site-specific recombination (Metzger and Chambon, 2001). Previous experiments based on recombination efficiency and CreER subcellular localization indicated that Cre recombinase activity peaks within $24 \mathrm{~h}$ and subsides $36-48 \mathrm{~h}$ after tamoxifen administration (Hayashi and McMahon, 2002). Therefore, interchromosomal recombination and consequent labeling of GCPs is limited to $\sim 1 \mathrm{~d}$ after tamoxifen administration. Application of $8 \mathrm{mg}$ of tamoxifen intraperitoneally allows us to maintain a low frequency of labeled cells ( $\sim 1.6$ cluster of GCs per half cerebellum). Statistical analyses suggest that each spatially restricted cluster of labeled GCs is derived from a single recombination event (Zong et al., 2005).

We fixed and stained the cerebella from animals at different ages (Fig. 1D) and quantified the total number of green and red cells within each $\mathrm{G}_{2}-\mathrm{X}$ clone (Fig. $1 C_{I^{-}}$ $\mathrm{C}_{2}$ ). Green and red cells were intermingled or segregated independent of type of clone (EGL or IGL). We find that most $\mathrm{G}_{2}-\mathrm{X}$ clones have similar numbers of red and green cells (Fig. 1D). This finding is inconsistent with an asymmetric model of cell division and supports the notion that at E17.5, or shortly afterward, GCPs predominantly undergo symmetric cell division, giving rise to two daughter cells with an equal potential of generating similar numbers of GC progeny. Although some clones are biased toward one color (e.g., $\mathrm{C} 15)$, they are the exception to the trend and may result from the occasional GCP cell death.

To test whether the division mode changes over time, we extended this analysis to four different postnatal times by applying tamoxifen at $\mathrm{P} 3, \mathrm{P} 6, \mathrm{P} 9$, and $\mathrm{P} 12$, while always examining the clones at P21 (Fig. 1E). At P21, GCP proliferation and GC differentiation are complete. At all injection time points, we find that most $\mathrm{G}_{2}-\mathrm{X}$ clones contain similar numbers of red and green cells within a clone (Fig. $1 E$ ). We conclude that at these postnatal times, GCPs also undergo predominantly symmetric division leading to exponential expansion of progeny number.

The exponential expansion of GCPs makes it possible that each GCP at E17.5 gives rise to a median of 250 GCs during a period of, at most, 3 weeks of expansion (see Fig. $4 \mathrm{~A}$ below). A pure linear 


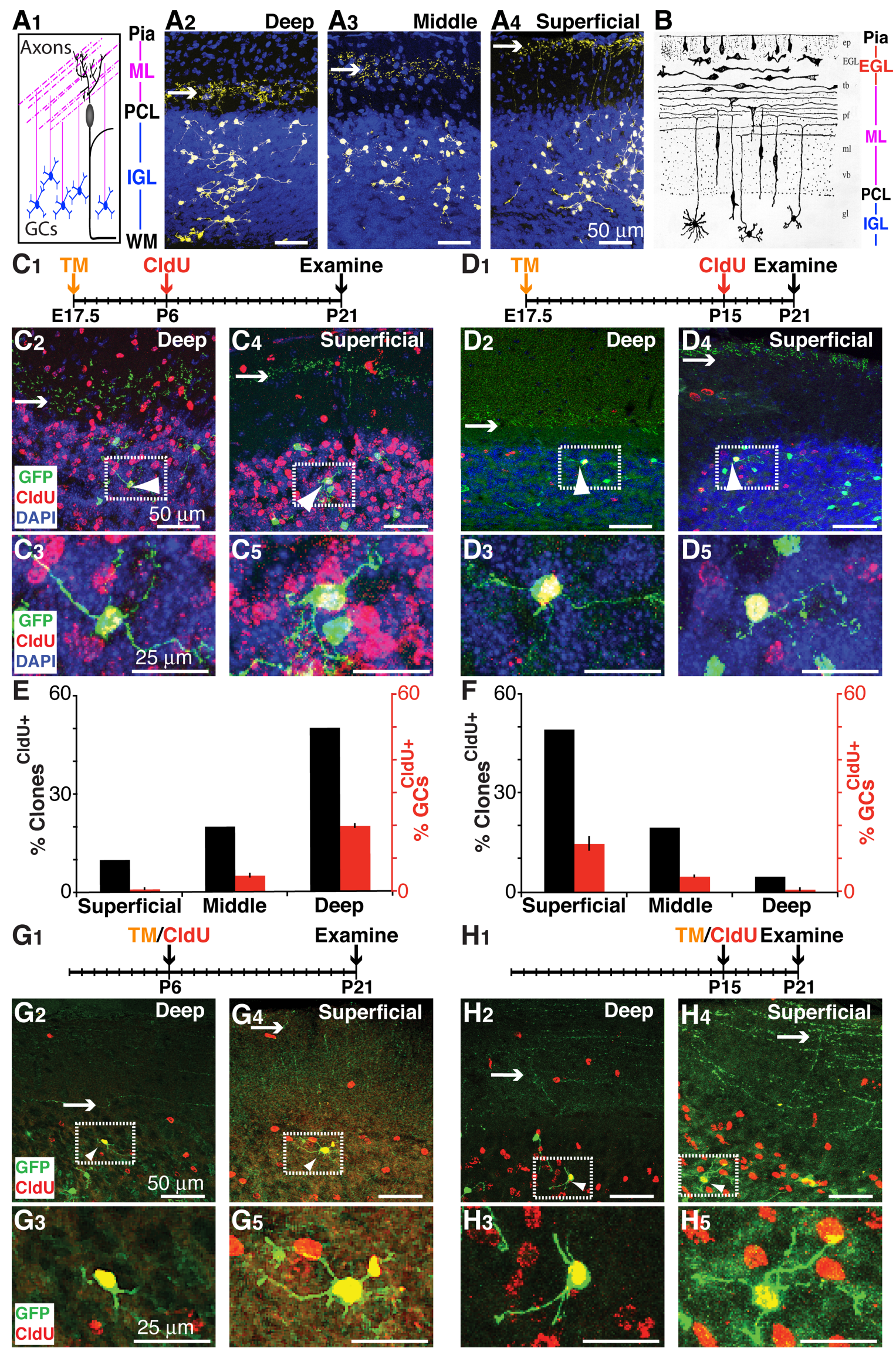


expan ${ }^{1}$ sion model requires the GCP cell cycle period to be $\sim 2 \mathrm{~h}$ (21 d $\times 24 \mathrm{~h} / \sim 250)$, much too fast for a mammalian neural precursor with a cell cycle period of at least $8 \mathrm{~h}$ (Caviness and Takahashi, 1995; Takahashi et al., 1995, 1997). The exponential model requires only eight cell divisions to expand from one labeled precursor to $\sim 250$ progeny $\left(2^{8}\right)$. If this were the case, the average length of the cell cycle would be $2.6 \mathrm{~d}$ ( $21 \mathrm{~d}$ per eight cell divisions), assuming that (1) there is minimal contribution by cell death and (2) all GCPs divide at the same rate all the time. Although assumption (1) is consistent with previous studies (Krueger et al., 1995; Muzumdar et al., 2007), assumption (2) does not apply (see below).

\section{Most GCs stack their axons within the ML in a temporal sequence}

We have previously conducted preliminary studies to examine the relationship between lineage and axon projection patterns of GCs (Zong et al., 2005). We labeled GCPs by administering tamoxifen at E17.5 and analyzed the GC progeny at P21. Interestingly, these experiments revealed that single GCPs at E17.5 give rise to GC progeny that project axons to a restricted sublayer in the ML despite the fact that their cell bodies disperse throughout the IGL (Fig. 2A) (Zong et al., 2005).

Classical studies suggest an orderly differentiation of GCs, which we coin the "stacking model" (Altman and Bayer, 1997). This hypothesis states that GCs that differentiate first leave their axons at the deepest sublayer in the ML; GCs that differentiate progressively later leave their axons in progressively more superficial sublayers in the ML. The supporting evidence for this hypothesis came primarily from developmental snapshots using Golgi staining: deepest projecting axons tend to belong to the most morphologically differentiated GCs (Fig. 2B) (Ramon y Cajal, 1911). If this stacking model were correct, our observation that clonally related GCs project their axons to specific sublayers would imply that (1) all clonally related GCs "synchronously" differentiate within a narrow time window during postnatal development and (2) the time windows for differentiation occur earlier for deeply projecting clones than for the superficially projecting clones.

To test these predictions, we first performed a set of experiments to quantitatively test the stacking model in mice. Because there is no correlation between the depth of GC body position within the GC layer and the depth of their axon position in the ML (Fig. 2A) (Zong et al., 2005), we need a method to correlate birth of individual GCs with their axonal positions.

In the first set of experiments, we injected tamoxifen at E17.5 to generate MADM clones and subsequently injected the thymidine analog CldU at P6 or P15 to label dividing cells in the S phase (Takahashi et al., 1992; Vega and Peterson 2005). We then analyzed P21 brains to determine the correlation of birth timing and axon projection of each MADM-labeled clone (Fig. $2 C_{1}, D_{1}$ ). Strong CldU-positive cells should be born shortly after injection time because there is no dilution of the label resulting from the lack of further cell divisions. The stacking model would predict that superficially projecting clones are composed of cells born exclusively at a late developmental time point (e.g., P15), whereas deeply projecting clones are composed of cells born exclusively at an early developmental time point (e.g., P6). For example, a deep projecting clone composed of cells that were born at P15 would be incompatible with a strict stacking model.

When CldU was injected at either P6 or P15, we found examples in which CldU-positive cells in MADM-labeled GC clones project axons to both superficial and deep layers (Fig. $2 C_{2}^{-}$ $C_{5}, D_{2}-D_{5}$ ). We quantified these events in two different ways. We took the position of the mean axonal projection of GC clones and bin into the deepest one-third, middle one-third, or most superficial one-third. First, we quantified the percentage of GC clones with at least one CldU-labeled cell for each type of clone (percentage of clones ${ }^{\mathrm{CldU}+}$ ) (Fig. 2 E, F, black). Second, we quantified the percentage of CldU-positive cells within these clones (percentage of GCs ${ }^{\mathrm{CldU}+}$ ) (Fig. 2 E, F, red). Both analyses gave similar results: deep projecting $\mathrm{GC}$ clones injected with $\mathrm{CldU}$ at $\mathrm{P} 15$ had a higher frequency of clones $(\sim 50 \%)$ and cells per clone $(\sim 20 \%)$ labeled with CldU. In contrast, when CldU was injected at P15, labeled cells are found with higher frequency in superficial projecting GC clones ( $\sim 50 \%$ of clones, $\sim 15 \%$ of cells per clone). Multiplying the percentage of clones that have at least one CldU-labeled cell by the average percentage of CldU-labeled cells per clone gives us a labeling index for the likelihood of labeling a class of GC with CldU. We find that CldU injection at P6 will label $\sim 0.1 \%(\sim 10 \%$ $\times \sim 1 \%)$ of superficial projecting GCs and $\sim 10 \%(\sim 50 \% \times$ $\sim 20 \%$ ), or 100-fold more, for deep projecting GCs. Conversely, injection of CldU at P15 will label $\sim 7.5 \%(\sim 50 \% \times \sim 15 \%)$ of superficial projecting GCs and $\sim 0.05 \%(\sim 5 \% \times \sim 1 \%)$, or 150 fold less, of deep projecting GCs. These results provide quantitative support for the stacking model. They also suggest that the stacking model is not absolute, because both P15 CldU-positive cells that project to deep layers and P6 CldU-positive cells that project to superficial layers exist, albeit at a very low frequency (see Discussion).

In a second set of experiments, we coinjected tamoxifen and CldU to induce MADM recombination and label dividing cells, respectively (Fig. $2 G_{1}, H_{1}$ ). Tamoxifen injection during early postnatal development results in MADM-labeled postmitotic and mitotic cells; however, CldU labeling allowed us to identify which of these GCs were born shortly after the time of CldU injection. CldU is diluted with each round of division, so cells born later may also be labeled, but less intensely. We quantified only strong CldU-positive cells, which are likely to be born shortly after CldU injection. We found that coinjection with ta-

Figure 2. GCsstack their axons in the ML in a temporal sequence from deep to superficial sublayers. $A$, To induce $M A D M G_{2}-X, G_{2}-Z$, or $G_{1}$ labeling, tamoxifen was applied intraperitoneally at E17.5 in the pregnant mother. Progeny of the genotype $G R / R G ; \beta$-Actin-CreER were examined. $A_{1}$, A schematic of cerebellar layers in the adult mouse. PCL, Purkinje cell layer; WM, white matter. $A_{2}-A_{4}$ Exemplary confocal images of MADM G or $\mathrm{G}_{2}$-Zlabeling of clones analyzed at $\mathrm{P} 21$, a time point when $\mathrm{GC}$ s have completed migration and exhibit mature morphology. All clonally related $\mathrm{GCS}$ project axons to the same restricted sublayer in the ML. For quantitative analysis in this and subsequent figures, we compartmentalize GC clones into one of three classes based on their average axonal projection pattern: deep (lower one-third), middle (middle one-third), superficial (top one-third). B, A schematic drawing by Ramon y Cajal (1911) depicts previous observations via Golgi staining, suggesting that GCs may stack their axons in accordance to their birth timing. $C_{1}$, A schematic of the experiment: tamoxifen application at E17.5, followed by a pulse injection of CldU at P6, and examination at P21. $\boldsymbol{C}_{2}, \boldsymbol{C}_{4}$, Exemplary confocal images of deep and superficial projecting $\mathrm{GC}$ clones, respectively. $\boldsymbol{C}_{3}, \boldsymbol{C}_{5}$, Magnified images of boxes in $\boldsymbol{C}_{2}$ and $\boldsymbol{C}_{4}$ demonstrating colabeling of $\mathrm{GFP}$ and CldU. D, Same as C, except that CldU was applied at P15. E, Percentage of IGL clones that have one or more cells labeled with CldU (Clones ${ }^{\text {CldU + }}$ ) shown in black and percentage of GCs in a given IGL clone that have $\mathrm{CldU}$ labeling ( $\mathrm{GCS} \mathrm{CldU}^{\mathrm{C}}$ ) shown in red for different types of IGL clones. Quantifications correspond to the timeline depicted in $\boldsymbol{C}_{1}$. $\boldsymbol{F}$, Same as $\boldsymbol{E}$, except quantifications correspond to the timeline depicted in $D_{1}, G_{1}$, A schematic of the experiment: coinjection of tamoxifen and CIdU at P6 and examination at P21. $G_{2}, G_{4}$, Singly labeled CldU $+/ G F P+G C s$ and their corresponding projection to the ML, deep and superficial, respectively. $\boldsymbol{G}_{\mathbf{3}}, \boldsymbol{G}_{\mathbf{5}}$, Magnified images of boxes in $\boldsymbol{G}_{\mathbf{2}}$ and $\boldsymbol{G}_{\mathbf{4}}$ demonstrating colabeling of $\mathbf{G F P}$ and CldU. $\boldsymbol{H}$, Same as $\boldsymbol{G}$, except that CldU was applied at P15. 
A1
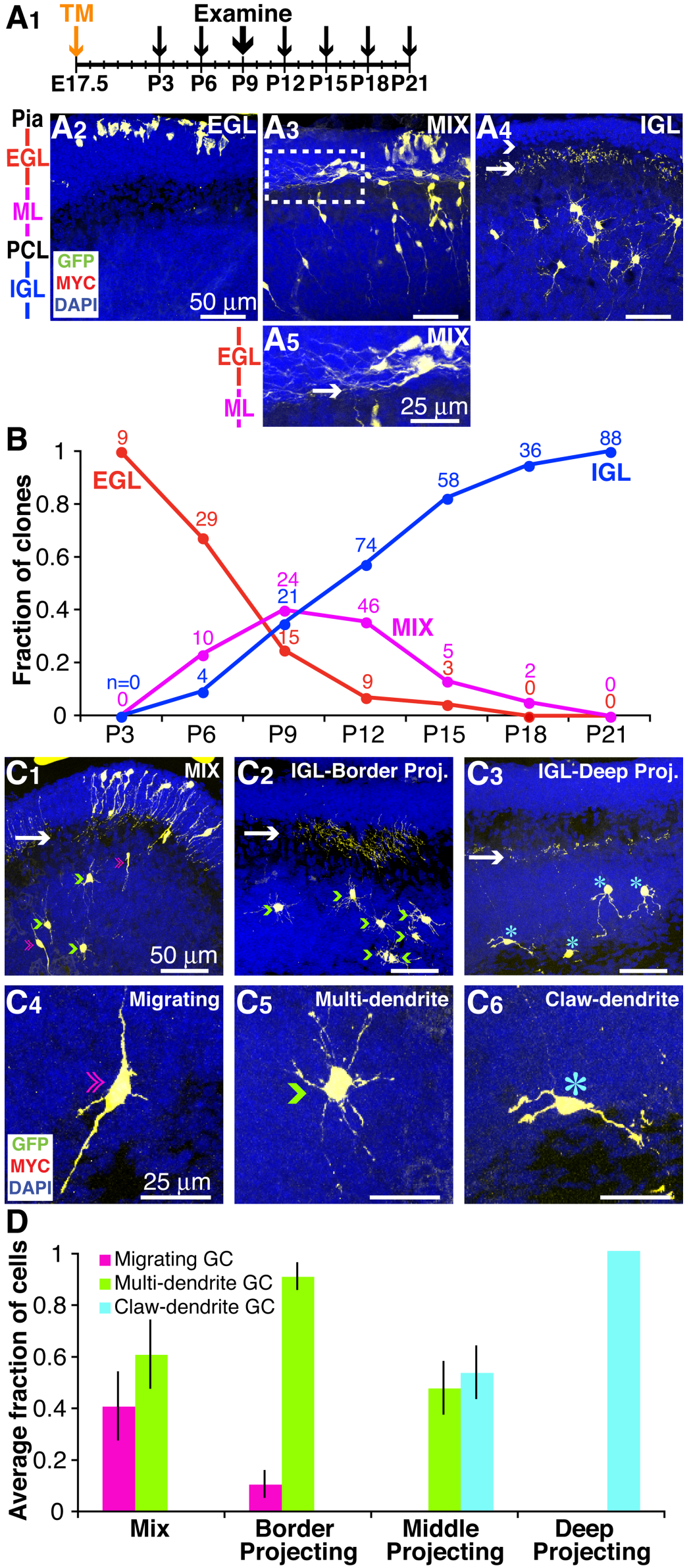

moxifen and CldU at P6 double labeled deep projecting GCs ( $n=20$ cells) (Fig. $\left.2 G_{2}, G_{3}\right)$ and coinjection with tamoxifen and CldU at P15 double labeled superficial projecting GCs $(n=22$ cells) (Fig. $\left.2 \mathrm{H}_{4}, \mathrm{H}_{5}\right)$. However, we found rare exceptions such as cells born at $\mathrm{P} 6$ projecting to superficial sublayers ( $n=4$ cells) (Fig. $\left.2 G_{4}, G_{5}\right)$ and cells born at P15 projecting to deep sublayers in the ML ( $n=2$ cells) (Fig. $3 \mathrm{H}_{2}, \mathrm{H}_{3}$ ). Consistent with the first experiment, the stacking model applies to most GCs, with notable exceptions.

\section{Clonally related GCs differentiate}

within a narrow time window in an orderly sequence

Having verified that the stacking model is mostly applicable, we next examined the hypothesis that clonally related GCs exit the cell cycle within a specific time window and thus project axons to a similar sublayer in the ML. We followed GCP expansion during postnatal development by generating GC clones at E17.5 and analyzing their progeny at different time points during development (Fig. $3 A_{1}$ ).

When examining MADM-labeled clones, we noted the position of cell bodies (EGL, ML, or IGL) using DAPI as a counterstain and the morphology of cells (polyhedral, nonpolyhedral, axonal, and dendritic processes). Examination of early postnatal time points (less than P4) revealed that all cells in all clones were located exclusively in the EGL (Fig. $3 A_{2}, B$ ), indicating that this is a period consisting predominantly of GCP expansion.

\footnotetext{
Figure 3. Orderly differentiation of $\mathrm{GC}$ clones during postnatal development. $A_{1}$, A schematic of the experiment: tamoxifen application at $E 17.5$, and dissection time points from P3 to P21. $A_{2}-A_{4}$, Confocal images illustrating three main classes of clones found at P9: EGL, MIX, and IGL. IGL clones always have axonal projections $\left(\boldsymbol{A}_{4}\right.$, arrow) in the ML positioned below the EGL/ML border $\left(\boldsymbol{A}_{4}\right.$, arrowhead). $\boldsymbol{A}_{5}$, Magnified image of the box in $\boldsymbol{A}_{3}$ depicting formation of $\mathrm{GC}$ axons (arrow) at the EGL/ML border and parallel to the pia typical of all MIX clones. $\boldsymbol{B}$, Frequency distribution of the three different types of clones illustrated in $A_{2}-A_{4}$ for different postnatal time points. $C_{1}-C_{3}$, Confocal images illustrating three classes of clones observed at $\mathrm{P} 12$ undergoing maturation of dendritic processes. Cells of a MIX clone have the least mature dendritic morphology, and IGL clones projecting to progressively deeper sublayers in the ML have progressively more mature dendritic morphology. $\boldsymbol{C}_{4}-\boldsymbol{C}_{6}$, Confocal images of three types of postmitotic GCs undergoing maturation of processes from migrating leading processes, to multiple dendrites, to clawlike dendrites. Colored symbols in $\boldsymbol{C}_{4}-\boldsymbol{C}_{6}$ correspond to $\mathrm{GC}$ types found in $\boldsymbol{C}_{1}-\boldsymbol{C}_{3}$. D, Frequency of dendritic maturation among $\mathrm{GCS}$ exemplified in $\boldsymbol{C}_{4}-\boldsymbol{C}_{6}$ for different classes of granule clones exemplified in $\boldsymbol{C}_{1}-\boldsymbol{C}_{3}$. Colored bars correspond to colored arrowheads and borders in $\boldsymbol{C}$.
} 

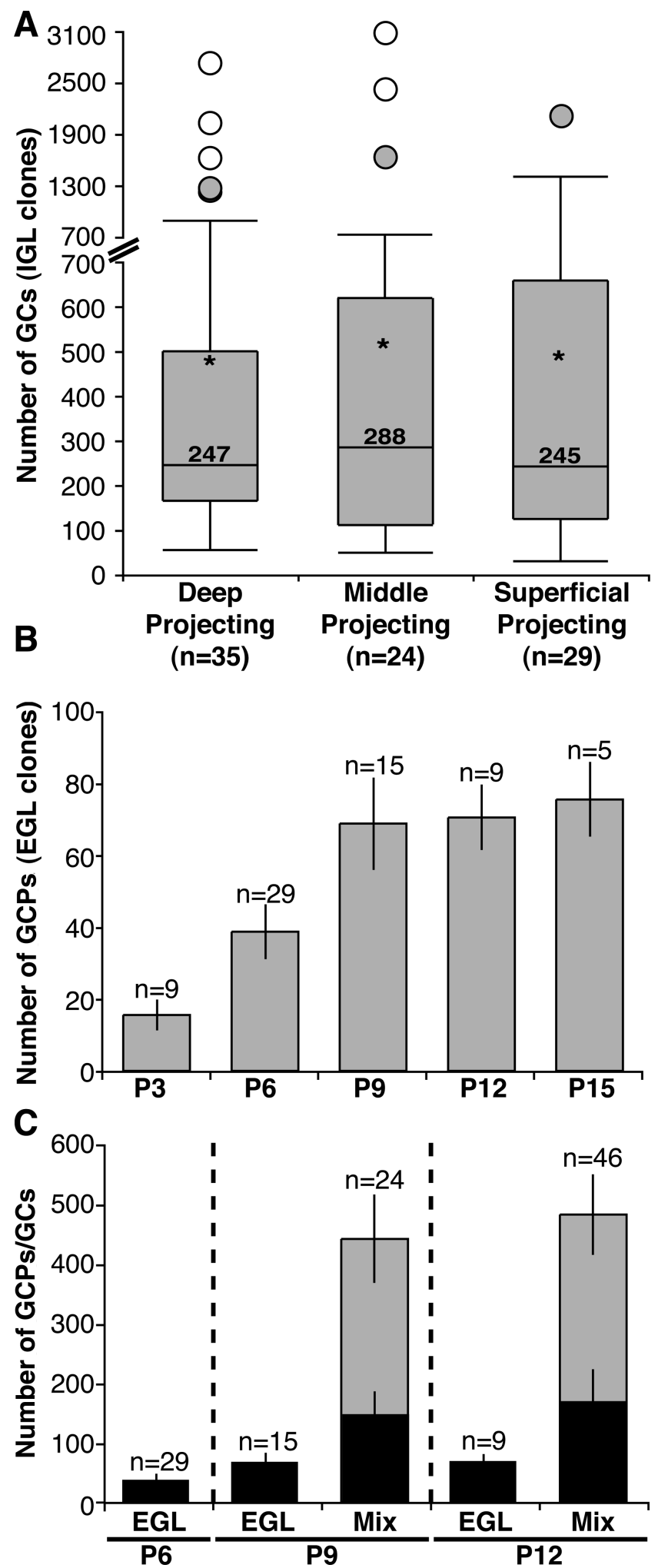

Figure 4. Cell number matching among $\mathrm{GC}$ clones with distinct axonal projection patterns. $\boldsymbol{A}$, Box plot representative of IGL clones grouped by the corresponding axonal projection pattern. The box demarcates the lower quartile, median (number), mean (asterisk), and upper quartile. Whiskers represent the lowest and highest values that are not outliers $[<1.5 \times$ interquartile range (IQR)]. Mild outliers are represented by a filled dot $\left(>1.5^{*} \mathrm{IQR}\right.$ and $<3 * I Q R)$, and extreme outliers are represented by an open $\operatorname{dot}(>3 * I Q R) . B$, Average number of GCPs per EGL clone quantified at different postnatal time points. $C$, Average number of GCPs per EGL clone compared with the average number of GCPs/GCs per MIX clone at P9. Light gray bars illustrate average numbers of cells in MIX clones. Black bars illustrate average numbers of cells in the EGL region of MIX clones (representing GCPs).
MADM clones analyzed at later postnatal time points (P6-P15) fell into three classes: class 1 (EGL), labeled cells are exclusively in the dividing EGL layer and characterized by a polyhedral shape (Fig. $3 A_{2}$ ); class 2 (MIX), labeled cell bodies are found in EGL, migratory route to IGL, and in IGL (Fig. $3 A_{3}$ ); class 3 (IGL), labeled cell bodies are predominantly in IGL (Fig. $3 A_{4}$ ).

Given the orderly organization of cells at different stages of differentiation during postnatal cerebellar development (Hanaway, 1967; Eccles, 1970; Herrup and Kuemerle, 1997), we interpret EGL clones to indicate that all labeled cells are still GCPs and none have undergone differentiation. IGL clones indicate that all labeled cells have already completed their division and migration through the ML to occupy their final destination in IGL; indeed, most labeled GCs in most IGL clones have elaborated their dendrites within the IGL. MIX clones are most likely caught in the process of differentiation: some cells have already completed their migration, some are in the process of migrating from EGL to IGL, and the rest are still dividing in the EGL. Interestingly, close examination of all MIX clones showed labeled axons extending at the ML/EGL border (Fig. $3 A_{5}$, arrow) as would be predicted by the stacking model. In contrast, all IGL clones examined from P6 to $\mathrm{P} 12$ project axons in sublayers below the ML/EGL border (Fig. $3 A_{4}$, arrow and arrowhead). By P21, all clones have cell bodies in the IGL, consistent with the end of morphological development of the cerebellar cortex. Quantification of the frequencies for each of three clonal types at different developmental stages reveals an orderly transition from EGL to MIX to IGL types as development proceeds (Fig. 3B).

The existence of EGL clones as late as P15 indicates that all progeny from these clones will differentiate after P15; the appearance of IGL clones as early as P6 indicates that all progeny from these clones have exited the cell cycle before P6. Together, these data rule out the possibility that all clonally related GCs differentiate throughout the entire period of postnatal neurogenesis and support the notion that clonally related GCs exit the cell cycle within specific time windows.

Because GC dendrites undergo a stereotyped maturation process from a single leading process (migrating) to multiple dendrites to few (three to seven) dendrites with claw-like terminal indicative of synapse maturation (Ramon y Cajal, 1911), we also used dendrite morphology to assess the GC differentiation status for different clonal types (Fig. $3 C_{1}-C_{6}$ ). We found that GCs that project axons deeper in the ML have more fully developed dendritic claws. In contrast, GCs from IGL clones that project axons near the ML/EGL border, and all MIX clones, have immature dendritic differentiation (Fig. 3D). These observations indicate that GCs belonging to the MIX clones are the most recently born and leave their axons at the most superficial part of the ML during these developmental snapshots (see above), providing further proof that GCs stack their axons in chronological order.

\section{Average expansion rate of GCPs gradually decreases during} postnatal development

Our findings provide evidence that GC clones that project deep in the ML must undergo differentiation earlier than GC clones that project superficial in the ML. One prediction from these findings is that clonally related "deep projecting" GCPs will have less time to divide compared with clonally related "superficial projecting" GCPs and therefore one would expect deep projecting GC clones to be smaller, on average, than superficial projecting GC clones. We sorted GC clones induced at E17.5 and examined at P21 into one of three groups according to their average axon projection depth in the ML: the bottom one-third (deep), middle one-third 
(middle), top one-third (superficial) (Fig. 2A). Next, we quantified the total number of GCs within each clone by counting the cell body number in consecutive sagittal sections that span the entire clone. Surprisingly, we found that the median of GCs per clone is similar regardless of whether their axon projection is to the deep, middle, or superficial sublayers in the ML (Fig. 4A). In all three subgroups, we find heterogeneous clone size, ranging from $<50$ to $>2000$ cells, with a median of $\sim 250$. The heterogeneity in clone size may be caused by occasional cell death of early GCPs, or a further heterogeneity of GCP proliferation potential within each subgroup. However, these differences are independent of the axon projection type, suggesting that time of cell cycle exit is not a predominant variable.

We set out to resolve this discrepancy: GC clones projecting axons to deep sublayers have less time to divide because they differentiate earlier in development; however, they are similar in number compared with GC clones projecting axons to superficial sublayers. We examined GCP expansion rates during postnatal development, because differences in expansion rates during development may partially explain this finding. We induced MADM clones at E17.5 and quantified the number of labeled cells per clone at different postnatal time points as shown in Figure $3 A$. We first examined EGL clones, which represent the stage of GCP division. We found that the expansion rate decreases as development proceeds (Fig. $4 B$ ). The average rate of GCP doubling is about once per day between E18 and P3, declines to once per $2 \mathrm{~d}$ from P3 to P6, and further declines afterward (Fig. $4 B)$. The progressively slow GCP expansion rate later in development therefore can contribute to, but does not by itself explain, the clone size discrepancy.

\section{Departure from the trend: GCPs speed up division shortly before the onset of GC differentiation}

The analysis in EGL clones represents an overall trend of GCP expansion but does not take into consideration that at any given time there could be different subpopulations of GCPs exhibiting different behavior. Indeed, when we include MIX clones into the cell expansion analysis, we found, strikingly, that MIX clones are much larger than EGL clones examined at the same developmental stage. Examples for two different stages of P9 and P12 are quantified in Figure 4C. Because all clones that contain differentiated cells derive from EGL clones at an earlier stage (e.g., EGL clones at P6 should give rise to EGL, MIX, and/or perhaps IGL clones at P9), these data suggest that shortly before onset of GC differentiation, GCPs expand faster to account for the differences between the cell numbers in EGL clones and those that contain differentiated cells. Interestingly, even the number of GCPs (counting only cells in EGL) in a MIX clone is larger than the number of GCPs in an EGL clone for the same developmental time point (Fig. $4 C$, compare black bars).

This increase in cell number expansion could be caused by a reduction in GCP death or an increase in cell proliferation. Because the contribution of cell death is not a major contributor to the decrease in expansion during postnatal development (see Discussion), it is more likely caused by increased cell proliferation or speedup of the GCP cell cycle. To experimentally test this hypothesis, we generated MADM clones by tamoxifen injection at E17.5 and determined the proportion of GFP cells labeled with a pulse of CldU (S phase marker) (Fig. $5 A_{1}$ ) or $\mathrm{pH} 3$ (M phase marker) (Fig. $5 C_{1}-C_{3}$ ). In general, the duration of the $S$ and $M$ phases has little variation (Cameron and Greulich, 1963; Prescott, 1968; Smith and Martin, 1973). Therefore, the percentage of cells in the S and M phases should reflect the cell cycle length: the
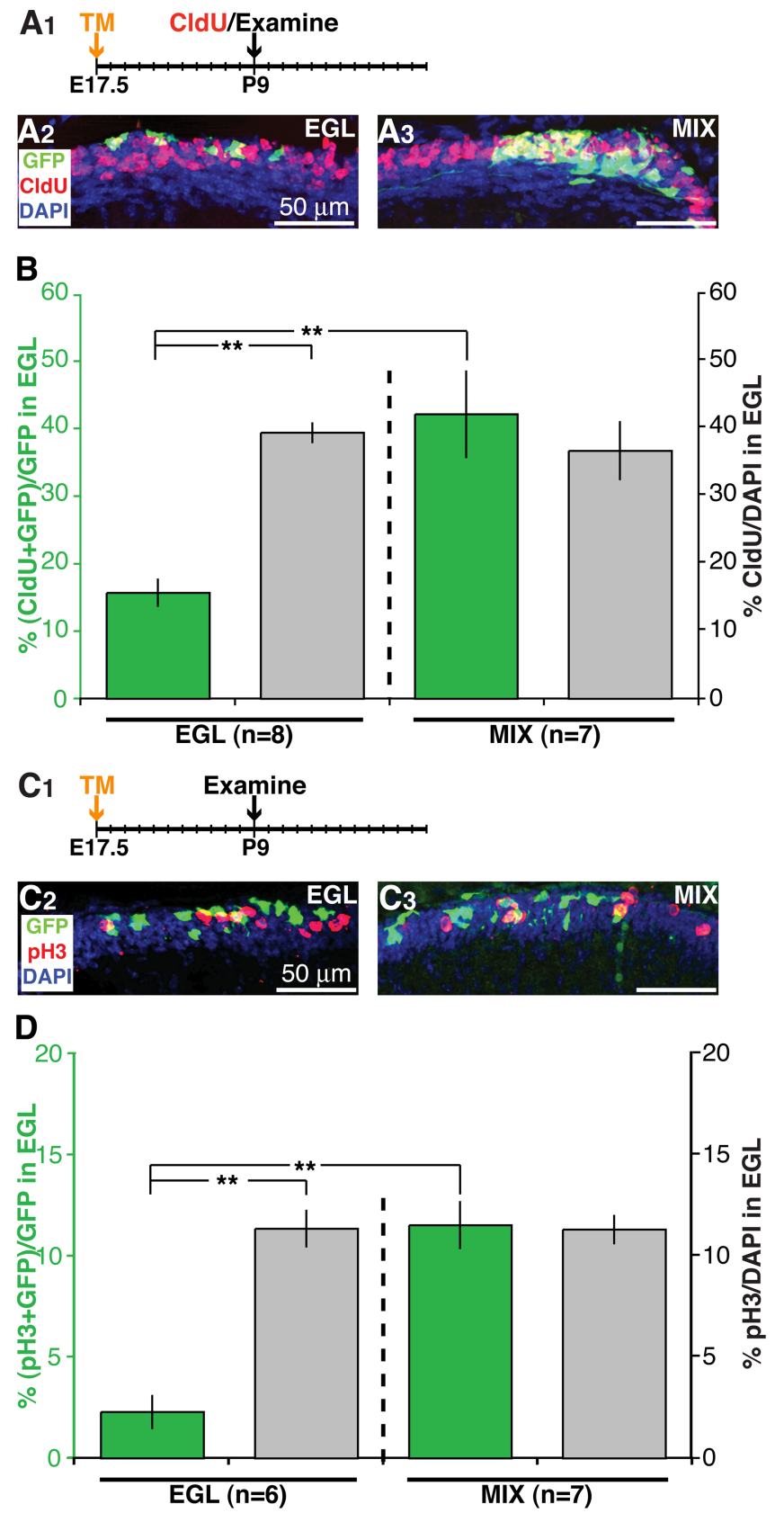

Figure 5. Clonally related GCPs divide faster shortly before differentiation. $\boldsymbol{A}_{\boldsymbol{1}}$, A schematic of the experiment: tamoxifen application at E17.5, followed by a pulse injection of $\mathrm{CldU}$ at $\mathrm{P} 9$ to label the $S$ phase. Mice were killed $2 \mathrm{~h}$ after CldU injection, and clonally related $\mathrm{GCS}$ were examined for colabeling with CIdU and their axonal projection pattern. $\boldsymbol{A}_{2}, \boldsymbol{A}_{\mathbf{3}}$, Representative confocal images of a P9 MADM-labeled EGL clone and a MIX clone, respectively. $\boldsymbol{B}$, Quantification of the average representation of CldU-labeled cells within individual MADM clones (green bars), or among the surrounding DAPI-labeled cells within the EGL (gray bars). EGL MADM clones are significantly different from the surrounding EGL region and MIX MADM clones $\left({ }^{* *} p<0.001\right) . C_{1}$, A schematic of the experiment: tamoxifen application at E17.5, followed by examination of clonally related progeny at $\mathrm{P} 9$ costained with $\mathrm{pH} 3$ to label cells in the $\mathrm{M}$ phase. $\boldsymbol{C}_{\mathbf{2}}, \boldsymbol{C}_{\mathbf{3}}$, Representative confocal images of a P9 MADM-labeled EGL clone and a MIX clone, respectively. $\boldsymbol{D}$, Same as $\boldsymbol{B}$, except $\mathrm{pH} 3$ was used instead of CldU.

higher the percentage, the faster the cell cycle. Figure 5, $A_{2}$ and $A_{3}$, show two representative confocal images of MADM-labeled clones examined $2 \mathrm{~h}$ after CldU was injected at P9. P9 was chosen to maximize the chance of having both EGL clones (Fig. $5 A_{2}$ ) and MIX clones containing labeled cells in EGL/ML/IGL (Fig. $5 A_{3}$ ). 


\section{Temporally regulated neurogenesis and differentiation}

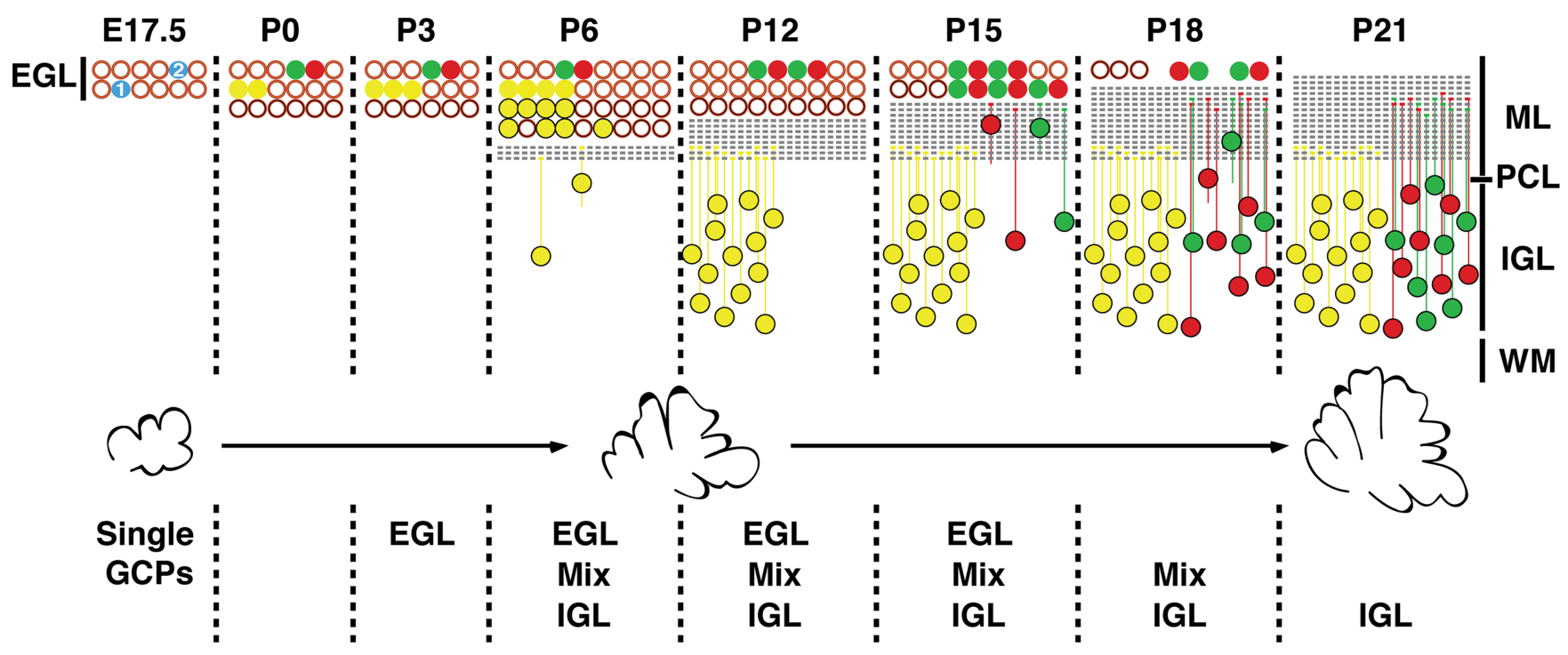

Figure 6. Schematic summary of neurogenesis and differentiation of clonally related GCs. We use two MADM clones induced at E17.5 to illustrate the main findings of this study: (1) the result of a $G_{1}$ or $G_{2}-Z$ recombination event, subsequently represented by yellow cells; (2) the result of a $G_{2}-X$ event, subsequently represented by green and red cells. GCPs undergo predominantly symmetric division during postnatal development (exemplified by same numbers of green and red cells). GCPs expansion decreases in the external granular layer (EGL) but speeds up division shortly before differentiation onset. At a specific temporal window, clonally related GCs exit the cell cycle and stack their axons in the molecular layer (ML) during differentiation. Early differentiating cells (yellow) stack their axons to deep sublayers in the ML, whereas cells that differentiate late (red and green) stack their axons to more superficial sublayers. This series of events produces GC clones that are distinct, each having a restricted axonal projection pattern, but that on average are similar in cell number. PCL, Purkinje cell layer; IGL, internal granular layer; WM, white matter.

As expected, CldU or pH3 does not label cells residing in the ML and IGL, because they have already exited the cell cycle.

Within the proliferative region of the outer EGL, we counted the number of CldU-positive or pH3-positive cells in each MADM clone. We divided these numbers by the total number of cells within that MADM clone to derive a percentage of cells within each clone that have been in the $S$ phase in the past $2 \mathrm{~h}$ or in the M phase at dissection time. Strikingly, MIX clones (containing cells in the EGL, ML, and IGL) display more than twice as many CldU-positive or four times as many $\mathrm{pH} 3$-positive cells in the EGL compared with EGL clones (Fig. $5 B, D$, green bars). As controls, we also quantified the percentage of CldU-positive or pH3-positive non-MADM-labeled cells within the EGL near each MADM clone, using the DNA dye DAPI as a nuclear marker. We find that $40 \%$ are CldU positive and $10 \%$ are $\mathrm{pH} 3$ positive for MADM-negative cells in the EGL region demarcated by the border of the MADM clone, regardless of whether the cell bodies are near EGL or MIX MADM clones (Fig. $5 B, D$, gray bars).

The above data provide direct evidence that EGL clones have a longer cell cycle compared with MIX clones, supporting a twophase model of GCP expansion: a slow phase while expanding exclusively within EGL and a fast phase close to the onset of differentiation. Furthermore, given that non-MADM-labeled EGL cells have the same percentage of CldU-positive (40\%) and pH3-positive (10\%) cells as MADM mix clones, the population of dividing cells in EGL at P9 are mostly consisting of those that will differentiate shortly. Thus, the total cell number derived from a single GCP at E17.5 may primarily be contributed by the rapid expansion phase regardless of the timing of cell cycle exit. This, in combination with the slow GCP cell cycle during most of the proliferation period within EGL (last section), can now explain the similar clone sizes regardless of the timing of cell cycle exit.

\section{Discussion}

Although the development of GCs has been studied extensively, our MADM-based analyses offer new insights into the rules that govern neurogenesis and differentiation of GCs, especially with regard to their relationship with lineage. Our findings are schematically summarized in Figure 6. GCPs undergo predominantly symmetric division and exponential expansion to generate large clusters of GCs. For a given E18 GCP, all progeny exit the cell cycle within a narrow temporal window and subsequently stack their axons at the most superficial layer of the developing ML. This narrow temporal window explains the phenomenon that GC clones project their axons to a specific sublayer in the ML (Zong et al., 2005). Our findings also suggest that although the average GCP proliferation in the EGL is progressively slower as development proceeds, there is a rapid expansion of clonally related GCPs shortly before differentiation. We discuss supporting evidence, molecular mechanisms, and functional implications.

\section{Clonally related GCs exit the cell cycle within narrow temporal windows}

The conclusion that clonally related GCs exit the cell cycle within a narrow temporal window originates from two observations: (1) clonally related GCs project their axons to a specific sublayer in the ML; and (2) GCs stack their axons in the most superficial layer at the time of their differentiation.

The stacking model was primarily inferred from developmental snapshots of Golgi samples (Altman and Bayer, 1997). Our results using combined CldU and MADM labeling support its generality. However, we observe some exceptions to the rule. The existence of occasional P6 CldU-positive GCs that project axons to superficial layers could be attributable to a very slow division rate, allowing for CldU to be retained in cells that differentiate at a much later stage. Also possible is that GCPs exit the cell cycle 
shortly after P6 but do not migrate until the rest of the clone starts to differentiate. Our finding that some P15 CldU-positive GCs are found in deep projecting clones suggests that these GCs must be born much later than their clonal siblings but somehow still "home" their axons to the appropriate corresponding sublayer.

Additional support for the stacking model, and the conclusion that clonally related GCPs exit the cell cycle within a narrow temporal window, comes from quantitative analysis of three clonal types. Comparing axonal projection patterns in MIX and IGL clones provides direct evidence that newborn GCs leave axonal projections at the border of EGL/ML. Closer observation of MIX and IGL clones shows a progression in dendritic maturation. Given that only EGL clones are present at P3, and the occasional IGL clone with mature dendrites are present at P6, the time window from the onset of cell cycle exit to completion can be within 3 d early in postnatal development.

\section{GCPs slow down expansion during postnatal development but undergo bursts of fast cell division shortly before differentiation}

Our finding that GC clones have a similar number of progeny regardless of their projection type appears to introduce a paradox. Two factors contribute to the resolution. First, after quantification of cell number in EGL clones, we find that GCPs progressively slow down their expansion during postnatal development, thereby likely minimizing discrepancies in cell number among early and late differentiating GC clones. Second, by comparing the cell number between EGL and MIX clones at P9, we find that GCPs undergo an increase in proliferation just before differentiation ensues. Indeed, more dividing cells are labeled with CldU and pH3 in MIX compared with EGL clones. This is likely attributable to a shortening in $G_{1}$ because we are more likely to obtain $\mathrm{G}_{2}$-X labeling in MIX (P9, 2 of 24; P12, 9 of 46) compared with EGL clones (P9, 0 of 15; P12, 0 of 9). Thus, GCPs in the EGL are heterogeneous, composed of slow and fast dividing populations. Previous studies have calculated the cell cycle period in the EGL to be 16-21 h from P1 to P15 (Fujita, 1967; Mares et al., 1970; Yoshioka et al., 1985). Our findings suggest that this rate of division is likely the average of the two subpopulations.

A possible mechanism we did not address is the contribution of apoptotic cell death. Although cell death in the EGL increases approximately fourfold from P7 to P14 (Krueger et al., 1995), $\sim 5.2 \%$ of all EGL cells die per day given a peak of $\sim 1.4 \%$ pyknotic cells and a duration of pyknoses of $\sim 6.5 \mathrm{~h}$ (Koppel et al., 1983), a number too small to account for reduced EGL clonal expansion as development proceeds. Moreover, cell death in the EGL is predominantly occurring at the border of the lower EGL and ML (Mares et al., 1970) and rarely in clusters (Smeyne and Goldowitz, 1989). Apoptotic cell death may also play a role if contributing preferentially in GCs that migrate late in development. However, the percentage of pyknotic cells in the IGL is less than in the EGL, decreases from P9 to P14, and is accounted for mostly by astrocytes (Krueger et al., 1995).

We note that the cell number among IGL clones is heterogeneous within each projection type, which may arise from differences in cell division and/or death. Given the low contribution of cell death and different EGL and MIX clones have similar percentages of CldU and/or pH3 labeling (Fig. 5), clonal heterogeneity likely arises from differences in the time spent in the fast dividing phase.

\section{Possible molecular mechanisms}

What determines a narrow differentiation time window for a particular GC clone? The signal may be intrinsically generated and/or extrinsically produced. Our findings resemble the observation of an intrinsic clock for oligodendrocyte differentiation in vitro (Temple and Raff 1986; Barres et al., 1994). When a single oligodendrocyte precursor is plated at clonal density (Hart et al., 1989), all progeny differentiate synchronously in response to exposure to thyroid hormone or mitogen withdrawal (Temple and Raff, 1986; Barres et al., 1994). The thyroid hormone receptor and cell cycle regulator $\mathrm{p} 57^{\mathrm{kip} 2}$ are potential intrinsic clock regulators (Barres et al., 1994; Dugas et al., 2007). Interestingly, an increase in $\mathrm{p} 57^{\mathrm{kip} 2}$ expression in oligodendrocyte precursor siblings is correlated with slowing down of division rate and cell cycle exit (Ghiani and Gallo, 2001; Dugas et al., 2007), similar to that seen in our analysis of GCPs in vivo. Additionally, hyperthyroidism leads to premature disappearance of the EGL and a corresponding reduction in GC number, whereas hypothyroidism prolongs EGL expansion and increases GC number (Legrand, 1986). It is possible that $\mathrm{p} 57^{\mathrm{kip} 2}$ and thyroid hormone might play an analogous function in regulating cell cycle exit of clonally related GCs.

A candidate extrinsic factor is Sonic hedgehog (Shh). Expression, loss- and gain-of-function studies support a model in which Purkinje cell-derived Shh regulates GCP proliferation (Wechsler-Reya and Scott, 1999; Lewis et al., 2004). Interestingly, there exists a constant ratio of $\sim 175$ GCs for each Purkinje cell (Wetts and Herrup, 1983), suggesting the GC number is regulated by the number and the length of time Purkinje cells secrete Shh. One of the transcriptional targets of Shh is Patched1 (Ptc1), which is itself a negative regulator of Shh signaling pathway (Goodrich et al., 1996; Marigo et al., 1996; Rohatgi et al., 2007). If the readout of an intrinsic clock is an essential component of Shh signaling pathway, then clonally related GCPs may acquire or augment Shh response at a certain developmental window so that their proliferation is enhanced. This could be followed by the negative-feedback regulation of the Shh signaling pathway via Ptcl expression, to ensure the termination of proliferation and the timely cell cycle exit of GCs.

\section{Functional implication for circuit formation and function}

Given the complex mechanisms needed to ensure that GC clones project axons to specific sublayers in the ML and are similar in cell number, we envision that this organization must have utility in the wiring and function of the cerebellar cortex. Each Purkinje cell receives input from both superficial and deep projecting GCs. However, deep and superficial projecting GCs predominantly synapse onto basket and stellate interneurons, respectively (Lemkey-Johnston and Larramendi, 1968). These interneurons send axons to distinct parts of the Purkinje cell and thus modulate Purkinje cell activity differentially. Additionally, GC axons decrease in diameter from deep to superficial (Fox and Barnard, 1957; Wyatt et al., 2005), which confers differences in information transfer. Finally, it takes the activation of many GC axons to activate a Purkinje cell (Bower and Woolston, 1983; Harvey and Napper, 1991). The dendritic integration theory predicts that axons synapsing onto a small portion of a dendritic tree would be more effective in inducing an action potential than if the same number of synapses are distributed widely (Migliore and Shepherd, 2002). Thus, if clonally related GCs were activated by a similar input, then such input would be most effective in driving Purkinje cell firing.

Different clonally related GCs may receive different mossy 
fiber inputs. DiI retrograde tracing has shown that mossy fibers from the vestibular ganglion reach the cerebellum at E13, the vestibular nuclei and spinal cord at E15, the lateral reticular nucleus and the pontine nuclei at $\mathrm{P} 0$, and the external cuneate nuclei postnatally (Berretta et al., 1991a,b; Ashwell and Zhang, 1992). Differences in the arrival times of mossy fibers can be used to form synapses with GCs that differentiate at different times. Thus, the properties of GC neurogenesis and differentiation we describe here could provide a mechanism that coordinates mossy fiber inputs to GCs and GC axonal output to different sublayers in the molecular layer.

\section{References}

Adams NC, Tomoda T, Cooper M, Dietz G, Hatten ME (2002) Mice that lack astrotactin have slowed neuronal migration. Development 129:965-972.

Alder J, Cho NK, Hatten ME (1996) Embryonic precursor cells from the rhombic lip are specified to a cerebellar granule neuron identity. Neuron 17:389-399.

Altman J, Bayer SA (1997) Development of the cerebellar system in relation to its evolution, structure and function. Boca Raton, FL: CRC.

Ashwell KW, Zhang LL (1992) Ontogeny of afferents to the fetal rat cerebellum. Acta Anat (Basel) 145:17-23.

Barres BA, Lazar MA, Raff MC (1994) A novel role for thyroid hormone, glucocorticoids and retinoic acid in timing oligodendrocyte development. Development 120:1097-1108.

Berretta S, Perciavalle V, Poppele RE (1991a) Origin of spinal projections to the anterior and posterior lobes of the rat cerebellum. J Comp Neurol 305:273-281.

Berretta S, Perciavalle V, Poppele RE (1991b) Origin of cuneate projections to the anterior and posterior lobes of the rat cerebellum. Brain Res 556:297-302.

Bower JM, Woolston DC (1983) Congruence of spatial organization of tactile projections to granule cell and Purkinje cell layers of cerebellar hemispheres of the albino rat: vertical organization of cerebellar cortex. J Neurophysiol 49:745-766.

Cameron IL, Greulich RC (1963) Evidence for an essentially constant duration of DNA synthesis in renewing epithelia of the adult mouse. J Cell Biol 18:31-40.

Caviness Jr VS, Takahashi T (1995) Proliferative events in the cerebral ventricular zone. Brain Dev 17:159-163.

Chenn A, McConnell SK (1995) Cleavage orientation and the asymmetric inheritance of Notch1 immunoreactivity in mammalian neurogenesis. Cell 82:631-641.

Dugas JC, Ibrahim A, Barres BA (2007) A crucial role for p57(Kip2) in the intracellular timer that controls oligodendrocyte differentiation. J Neurosci 27:6185-6196.

Eccles JC (1970) Neurogenesis and morphogenesis in the cerebellar cortex. Proc Natl Acad Sci USA 66:294-301.

Fox CA, Barnard JW (1957) A quantitative study of the Purkinje cell dendritic branchlets and their relationship to afferent fibres. J Anat 91:299-313.

Fujita S (1967) Quantitative analysis of cell proliferation and differentiation in the cortex of the postnatal mouse cerebellum. J Cell Biol 32:277-287.

Gage FH, Coates PW, Palmer TD, Kuhn HG, Fisher LJ, Suhonen JO, Peterson DA, Suhr ST, Ray J (1995) Survival and differentiation of adult neuronal progenitor cells transplanted to the adult brain. Proc Natl Acad Sci USA 92:11879-11883.

Ghiani C, Gallo V (2001) Inhibition of cyclin E-cyclin-dependent kinase 2 complex formation and activity is associated with cell cycle arrest and withdrawal in oligodendrocyte progenitor cells. J Neurosci 21:1274-1282.

Goodrich LV, Johnson RL, Milenkovic L, McMahon JA, Scott MP (1996) Conservation of the hedgehog/patched signaling pathway from flies to mice: induction of a mouse patched gene by Hedgehog. Genes Dev 10:301-312.

Guo C, Yang W, Lobe CG (2002) A Cre recombinase transgene with mosaic, widespread tamoxifen-inducible action. Genesis 32:8-18.

Hallonet ME, Teillet MA, Le Douarin NM (1990) A new approach to the development of the cerebellum provided by the quail-chick marker system. Development 108:19-31.
Hanaway J (1967) Formation and differentiation of the external granular layer of the chick cerebellum. J Comp Neurol 131:1-14.

Hart IK, Richardson WD, Heldin CH, Westermark B, Raff MC (1989) PDGF receptors on cells of the oligodendrocyte-type-2 astrocyte (O-2A) cell lineage. Development 105:595-603.

Harvey RJ, Napper RM (1991) Quantitative studies on the mammalian cerebellum. Prog Neurobiol 36:437-463.

Hatten ME, Heintz N (1995) Mechanisms of neural patterning and specification in the developing cerebellum. Annu Rev Neurosci 18:385-408.

Hayashi S, McMahon AP (2002) Efficient recombination in diverse tissues by a tamoxifen-inducible form of Cre: a tool for temporally regulated gene activation/inactivation in the mouse. Dev Biol 244:305-318.

Herrup K, Kuemerle B (1997) The compartmentalization of the cerebellum. Annu Rev Neurosci 20:61-90.

Ito M (1984) The modifiable neuronal network of the cerebellum. Jpn J Physiol 34:781-792.

Koppel H, Lewis PD, Patel AJ (1983) Cell death in the external granular layer of normal and undernourished rats: further observations, including estimates of rate of cell loss. Cell Tissue Kinet 16:99-106.

Krueger BK, Burne JF, Raff MC (1995) Evidence for large-scale astrocyte death in the developing cerebellum. J Neurosci 15:3366-3374.

Langman J, Guerrant RL, Freeman BG (1966) Behav of neuro-epithelial cells during closure of the neural tube. J Comp Neurol 127:399-411.

Legrand J (1986) Thyroid hormone effects on growth and development. New York: Marcel Dekker.

Lemkey-Johnston N, Larramendi LM (1968) Types and distribution of synapses upon basket and stellate cells of the mouse cerebellum: an electron microscopic study. J Comp Neurol 134:73-112.

Lewis PM, Gritli-Linde A, Smeyne R, Kottmann A, McMahon AP (2004) Sonic hedgehog signaling is required for expansion of granule neuron precursors and patterning of the mouse cerebellum. Dev Biol 270:393-410.

Machold R, Fishell G (2005) Math1 is expressed in temporally discrete pools of cerebellar rhombic-lip neural progenitors. Neuron 48:17-24.

Mares V, Lodin Z, Srajer J (1970) The cellular kinetics of the developing mouse cerebellum. I. The generation cycle, growth fraction and rate of proliferation of the external granular layer. Brain Res 23:323-342.

Marigo V, Davey RA, Zuo Y, Cunningham JM, Tabin CJ (1996) Biochemical evidence that patched is the Hedgehog receptor. Nature 384:176-179.

Martin AH (1967) Significance of mitotic spindle fibre orientation in the neural tube. Nature 216:1133-1134.

Mathis L, Nicolas JF (2003) Progressive restriction of cell fates in relation to neuroepithelial cell mingling in the mouse cerebellum. Dev Biol 258:20-31.

Metzger D, Chambon P (2001) Site- and time-specific gene targeting in the mouse. Methods 24:71-80.

Miale IL, Sidman RL (1961) An autoradiographic analysis of histogenesis in the mouse cerebellum. Exp Neurol 4:277-296.

Migliore M, Shepherd GM (2002) Emerging rules for the distributions of active dendritic conductances. Nat Rev Neurosci 3:362-370.

Muzumdar MD, Luo L, Zong H (2007) Modeling sporadic loss of heterozygosity in mice by using mosaic analysis with double markers (MADM). Proc Natl Acad Sci USA 104:4495-4500.

Prescott DM (1968) Regulation of cell reproduction. Cancer Res 28:1815-1820.

Rakic P (1971) Neuron-glia relationship during granule cell migration in developing cerebellar cortex. A Golgi and electronmicroscopic study in Macacus rhesus. J Comp Neurol 141:283-312.

Ramon y Cajal S (1911) Histology of the nervous system of man and vertebrates. Oxford: Oxford UP, 1995 translation.

Rohatgi R, Milenkovic L, Scott MP (2007) Patched1 regulates hedgehog signaling at the primary cilium. Science 317:372-376.

Ryder EF, Cepko CL (1994) Migration patterns of clonally related granule cells and their progenitors in the developing chick cerebellum. Neuron 12:1011-1028.

Sgaier SK, Millet S, Villanueva MP, Berenshteyn F, Song C, Joyner AL (2005) Morphogenetic and cellular movements that shape the mouse cerebellum; insights from genetic fate mapping. Neuron 45:27-40.

Sillitoe RV, Joyner AL (2007) Morphology, molecular codes, and circuitry produce the three-dimensional complexity of the cerebellum. Annu Rev Cell Dev Biol 23:549-577.

Smeyne RJ, Goldowitz D (1989) Development and death of external granu- 
lar layer cells in the weaver mouse cerebellum: a quantitative study. J Neurosci 9:1608-1620.

Smith JA, Martin L (1973) Do cells cycle? Proc Natl Acad Sci USA 70:1263-1267.

Takahashi T, Nowakowski RS, Caviness Jr VS (1992) BUdR as an S-phase marker for quantitative studies of cytokinetic behaviour in the murine cerebral ventricular zone. J Neurocytol 21:185-197.

Takahashi T, Nowakowski RS, Caviness Jr VS (1995) The cell cycle of the pseudostratified ventricular epithelium of the embryonic murine cerebral wall. J Neurosci 15:6046-6057.

Takahashi T, Nowakowski RS, Caviness Jr VS (1997) The mathematics of neocortical neuronogenesis. Dev Neurosci 19:17-22.

Temple S, Raff MC (1986) Clonal analysis of oligodendrocyte development in culture: evidence for a developmental clock that counts cell divisions. Cell 44:773-779.

Vega CJ, Peterson DA (2005) Stem cell proliferative history in tissue revealed by temporal halogenated thymidine analog discrimination. Nat Methods 2:167-169.

Wang VY, Zoghbi HY (2001) Genetic regulation of cerebellar development. Nat Rev Neurosci 2:484-491.

Wang VY, Rose MF, Zoghbi HY (2005) Math1 expression redefines the rhombic lip derivatives and reveals novel lineages within the brainstem and cerebellum. Neuron 48:31-43.

Wechsler-Reya RJ, Scott MP (1999) Control of neuronal precursor proliferation in the cerebellum by Sonic Hedgehog. Neuron 22:103-114.

Wetts R, Herrup K (1983) Direct correlation between Purkinje and granule cell number in the cerebella of lurcher chimeras and wild-type mice. Brain Res 312:41-47.

Wyatt KD, Tanapat P, Wang SS (2005) Speed limits in the cerebellum: constraints from myelinated and unmyelinated parallel fibers. Eur J Neurosci 21:2285-2290.

Yoshioka H, Mino M, Morikawa Y, Kasubuchi Y, Kusunoki T (1985) Changes in cell proliferation kinetics in the mouse cerebellum after total asphyxia. Pediatrics 76:965-969.

Zhang L, Goldman JE (1996) Developmental fates and migratory pathways of dividing progenitors in the postnatal rat cerebellum. J Comp Neurol 370:536-550.

Zhong W, Feder JN, Jiang MM, Jan LY, Jan YN (1996) Asymmetric localization of a mammalian numb homolog during mouse cortical neurogenesis. Neuron 17:43-53.

Zong H, Espinosa JS, Su HH, Muzumdar MD, Luo L (2005) Mosaic analysis with double markers in mice. Cell 121:479-492. 